\section{How to prepare students for life in practice}

\section{Mary Fraser}

Edinburgh Napier University, Edinburgh, UK

Stress is a recognised problem within the veterinary profession. Recent research by RCVS indicated that most veterinary nurses thought the job was stressful. The aim of this research was to determine how veterinary nursing students should be taught about the realities of practice.

An online questionnaire containing a mixture of closed and open questions was made available from January March 2015; only RVNs working in general practice for at least 6 months could take part. Ethical approval was granted by the supporting university's ethics committee.

A total of 151 responses were received to the question 'Do you think life in practice should be taught as a subject in its own right, or within another subject such as ethics or animal welfare?'

Thematic analysis was carried out manually and themes identified - namely that students need to be prepared for the reality of practice; that a taught module would be beneficial; and that time spent experiencing practice is important.

Deeper analysis revealed that students need to have an understanding of the requirements of practice, be able to cope with physical, technical and emotional aspects of practice, be aware of support that is available to them and have realistic expectations of what life in practice will be like.

Most respondents (105/151) felt that life in practice could be taught to students as a specific subject, covering stress management, putting professionalism into context and using scenarios, but only if the lecturers had experience of practice and were honest in their teaching. Others (33/151) felt that the reality of practice should be taught within other subjects such as ethics as it would ensure that students attended, did not think the subject boring and could see the relevance to their chosen career path.

Time in practice was regarded as important as placements allow students to deal with people and see real life, but some felt that they had been cushioned from reality as a student. They also felt that reflection or group discussions on time spent in practice was important.

In conclusion more time should be spent on training to prepare students in how to care for their own welfare as well as that of the animals.

\section{Is it "a wonderful life"? How well do we assess quality of life in cats?}

\section{Hannah Doit', Marnie Brennan', Richard Emes ${ }^{2}$, Rachel Dean'}

\footnotetext{
1 Center for Evidence-based Veterinary Medicine, University of Nottingham, Leicestershire, UK

2 Advanced Data Analysis Centre, University of Nottingham, Leicestershire, UK
}

Quality of life and wellbeing are concepts frequently used by veterinary surgeons and cat owners to assess efficacy and acceptability of treatments, case prognosis and overall cat wellbeing. This is usually done either by using tools designed for the purpose, or by subjective clinical assumptions of whether or not the cat is "doing well" in the opinion of the owner and vet. Using validated tools to assess quality of life and wellbeing of cats could allow vets and owners to have consistency and confidence in their decision making.

There are a number of published quality of life assessment tools for cats. The aim of this work was to systematically find and appraise the peer reviewed published quality of life tools for cats.

A structured search using the OVID interface via the
Medline and CAB Abstracts databases was performed in August 2015, to identify manuscripts containing keywords or subject headings related to cats and wellbeing or quality of life. The search results were then sorted using EndNote and inclusion and exclusion criteria were applied.

In total 917 unique papers were found, of which 166 met the inclusion criteria as they either stated that quality of life or wellbeing was assessed within the paper, or contained reference to quality of life or wellbeing within the abstract. A range of quality of life assessments were carried out within the papers, including questionnaires, scales and some unique named tools. However, not all of the tools described in the publications had been validated or were unique. In some publications, statements were made about an improvement in quality of life or wellbeing, without objective assessment using any form of tool.

As veterinary professionals we have a duty to ensure the welfare of our patients. Validated tools designed to assess quality of life are a valuable asset when taking an evidencebased approach to ascertaining the quality of life and wellbeing of cats.

If consensus can be reached on which quality of life tools should be included to assess quality of life in future studies, then the results of clinical studies will be more directly comparable. This would strengthen the evidence base and increase our knowledge of how to provide the best care and treatments to our feline patients. 